\title{
PLANAR GROUPS OF AUTOMORPHISMS OF STABLE PLANES
}

Markus Stroppel

(Semi-) planar groups of stable planes are introduced, and information about their size and their structure is derived. A special case are the stabilizers of quadrangles in compact connected projective planes (i.e. automorphism groups of locally compact connected ternary fields).

\section{INTRODUCTION.}

A stable plane $\mathrm{M}=(M, \mathcal{M})$ is a non-degenerate linear space, where the point space $M$ and the line space $\mathcal{M}$ carry locally compact Hausdorff topologies such that joining of points and intersection of lines are continuous operations. Moreover, intersection is stable (i.e. it has an open domain of definition), and the point space $M$ is assumed to have positive finite covering dimension. According to a deep result of $R$. Löwen [11], the covering dimension $\operatorname{dim} M$ is one of the integers $2,4,8$, or 16 . See e.g. [9] for basic facts and [11] for topological properties of stable planes (Our notion of "stable plane" is equivalent to "stabile lp-Ebene" in [9]).

Let Aut (M) denote the group of continuous collineations of $M$, endowed with the compactopen topology derived from the action on $M$. Automorphism groups of stable planes of higher dimension may effectively be studied via considering their action on invariant subplanes (if such subplanes exist). For this purpose, information about the kernel of such a restriction is required. This leads to the study of planar groups:

(1.1) DEFINITION.

a) A (closed) subgroup $\Lambda$ of Aut (M) is called semi-planar, if the set Fix $(\Lambda)$ of fixed points of $\Lambda$ carries a non-degenerate, non-discrete subplane of $M$. If $\operatorname{dim} \operatorname{Fix}(\Lambda)>0$, then $\Lambda$ is called planar.

b) An automorphism $\alpha$ is called (semi-) planar if the group generated by $\alpha$ is (semi-) planar.

The notion of subplane will be defined precisely in section 3 . 


\section{(1.2) REMARKS.}

a) It may be conjectured that non-discrete subplanes of dimension 0 do not occur. At present, this has only been proved for the case where $\operatorname{dim} M \leq 4$ (see [9: 1.34]).

b) In the case of compact connected projective planes, the results in this paper remain valid for each stabilizer of a quadrangle, although the quadrangle might generate a discrete subplane. Wherever the assumption of a non-discrete set of fixed points is needed in this paper, it can be replaced by the assumption that $M$ is projective. Note that the stabilizer of a quadrangle in a projective plane is the automorphism group of the corresponding ternary field. Thus our results may be interpreted for automorphism groups of locally compact connected ternary fields.

NOTATION. We denote the line joining the points $x$ and $y$ by $x y$, and the intersection point of lines $X$ and $Y$ by $X \wedge Y$. Exponential notation is used for the action of Aut (M) on $M$ and $\mathcal{M}$.

ACKNOWLEDGEMENTS. The main source of inspiration has been the treatment of stabilizers of quadrangles in compact connected projective planes by H. Salzmann [20: section 2], [21]. The author was introduced to the study of stable planes by H. Salzmann and R. Löwen, and also owes thanks to R. Bödi and T. Grundhöfer for helpful comments and encouragement.

\section{AUTOMORPHISM GROUPS.}

For each stable plane $M$, let Aut (M) denote the group of all continuous collineations, endowed with the compact-open topology (with respect to the action on the point space). According to [9: 2.9], the group Aut (M) is a locally compact, separable topological group. Let $\Lambda$ be a locally compact connected group. By the Malcev-Iwasawa theorem, the (covering) dimension of $\Lambda$ is finite if and only if a maximal compact subgroup of $\Lambda$ has finite dimension. For semi-planar groups, we prove this property in this paper. Since a semiplanar group can be obtained by taking the stabilizer of some quintangle (see [9: 1.34]), Halder's dimension formula [8] may be used to show that the dimension of Aut (M) is finite.

Each locally compact connected group $\Lambda$ is an inverse limit of Lie groups in the sense that there are compact normal subgroups $Z_{i}$ such that the factor groups $\Lambda / Z_{i}$ are Lie groups, $\mathrm{Z}_{i} \geq \mathrm{Z}_{i+1}$ and $\bigcap_{i \in \mathbb{N}} \mathrm{Z}_{i}=\mathbb{1}$. If $\operatorname{dim} \Lambda$ is finite, we can choose the $\mathrm{Z}_{i}$ such that $\operatorname{dim} \mathrm{Z}_{i}=0$. Then all the $\Lambda / Z_{i}$ are locally isomorphic, and $Z_{i}$ lies in the center of $\Lambda$. In this case, all the groups $\Lambda / \mathrm{Z}_{i}$ have the same Lie algebra $L$, and we call $\Lambda$ of type $L$. We use the notation of Tits [25] for the simple real and complex Lie algebras. An asterisk ("*") will be used e.g. in $A_{n}^{*}$ to denote an arbitrary real form of the complex Lie algebra $A_{n}$.

For a semi-simple compact lie group $\Phi$, there are only a finite number of isomorphism types of Lie groups that are locally isomorphic with $\Phi$. This may be used to derive the fact that $\Lambda$ contains a compact (hence closed) subgroup locally isomorphic with $\Phi$ if any of the $\Lambda / \mathrm{Z}_{i}$ contains the compact semi-simple group $\Phi$. Note also that connected subgroups of $\Lambda$ centralize each other if and only if their images in $\Lambda / Z_{i}$ have this property.

The classification of the real and complex almost simple Lie groups and their Lie algebras will be used without explicit quotations. The tables in [15] or [25] may serve as a source 
for the information required.

\section{SUBPLANES.}

Let $E \subseteq M$ be a set of points, and let $\mathbb{E}=(E, \mathcal{E})$ be the geometry induced on $E$, where $\mathcal{E}$ is the set of lines that are incident with at least two points of $E$. The geometry $E$ is called a full subplane if $E$ contains a quadrangle and each point that lies on two lines of $\mathcal{E}$ belongs to $E$.

Let $X \subseteq M$ be a set of points containing a quadrangle, and let $\mathbb{E}=(E, \mathcal{E})$ be the smallest full subplane of $M$ such that $X \subseteq E$ and $E$ is closed in $M$. Then $\langle X\rangle=E$ is called the subplane generated by $X$ (in M). The set $E$ is the closure of $\tilde{E}$, where $\tilde{E}=(\tilde{E}, \tilde{\mathcal{E}}$ ) is the smallest full subplane of $\mathrm{M}$ such that $X \subseteq \tilde{E}$. The set $\tilde{E}$ is obtained by the following procedure: let $X_{0}=X$, and $\mathcal{X}_{i}=\left\{x y \mid x, y \in X_{i}\right\}, X_{i+1}=\left\{x \mid x=G \wedge H, G, H \in \mathcal{X}_{i}\right\}$ for $i \geq 0$. Then $\tilde{E}=\bigcup_{i \geq 0} X_{i}$. This yields the implications

$$
\begin{aligned}
& X^{\alpha}=X \quad \Longrightarrow \quad \tilde{E}^{\alpha}=\tilde{E} \quad \Longrightarrow \quad E^{\alpha}=E \\
& \left.\alpha\right|_{X}=\left.\mathbb{1} \Longrightarrow \alpha\right|_{\tilde{E}}=\left.\mathbb{1} \Longrightarrow \alpha\right|_{E}=\mathbb{1}
\end{aligned}
$$

for any (continuous) automorphism $\alpha$ of M. In particular, the geometry induced on Fix $(\Lambda)$ is a full closed subplane for each semi-planar group $\Lambda$.

(3.2) REMARK. Note that, for each subset $X \subseteq M$, the restriction map $\left.\delta \mapsto \delta\right|_{X}$ is continuous with respect to the compact-open topologies on $\mathcal{C}(M, M)$ and $\mathcal{C}(X, M)$, cf. e.g. [13: $§ 7-6$, Ex. 1, p. 289]. In general, however, this map need not be open or closed. If, in particular, the set $X$ is $\Delta$-invariant for some (closed) subgroup $\Delta$ of Aut (M), then the group $\left.\Delta\right|_{X}$ need not be closed in Aut $(\langle X\rangle)$, and $\Delta \mid X$ need not be topologically isomorphic with the factor group $\Delta / K$, where $K$ is the kernel of the restriction. The continuity of the restriction map, however, yields the following information about the closure $\Upsilon$ of $\left.\Delta\right|_{X}$ in $\operatorname{Aut}(\langle X\rangle)$ :

- If $\Delta / \mathrm{K}$ is compact, then $\left.\Delta\right|_{X}$ is compact and hence closed in Aut $(\langle X\rangle)$. Consequently, the groups $\Delta / \mathrm{K}$ and $\Delta / X$ are topologically isomorphic.

- If $\Delta / K$ is connected, then $\Upsilon$ is connected [6: $V, 1.6]$.

- $\operatorname{dim} \Delta / \mathrm{K}=\left.\operatorname{dim} \Delta\right|_{X} \leq \operatorname{dim} \Upsilon$ (apply [14: III.6] to a compact neighbourhood in $\Delta / \mathrm{K}$ ).

- The factor group $\Delta / K$ is abelian, nilpotent, or solvable if and only if the group $\Upsilon$ has the property in question.

- If $\Delta / \mathrm{K}$ and $\Upsilon$ are Lie groups, then $\left.\Delta\right|_{X}$ is an analytical subgroup of $\Upsilon$. In particular, the Levi complement of $\left.\Delta\right|_{X}$ is contained in the Levi complement of $\Upsilon$ (cf. [26: 2.7.3 and 2.11.2]).

Further information about the topologies that occur with restrictions is comprised in section 4. We collect some useful general information about subplanes first.

We have the following maximum property for full closed subplanes of positive dimension: (3.3) LEMMA. Let $\mathrm{E}=(E, \mathcal{E})$ be a full closed subplane of $\mathrm{M}$ with $\operatorname{dim} E>0$. If $E \subset F$ and $\mathbb{F}=(F, \mathcal{F})$ is a closed subplane, then $\operatorname{dim} F>\operatorname{dim} E$. 
Proof. Assume that $\operatorname{dim} E=\operatorname{dim} F$. According to [11: Th. 11c)], $E$ is open in $F$. For any point $z \in F$, choose points $x, y \in E$ such that $y \notin x z$. Each of the lines $x z, y z$ meets the open set $E$ in more than one point. Thus $x z$ and $y z$ belong to $\mathcal{E}$ and hence $z \in E$, since $\mathrm{E}$ is full.

In a projective plane, any quadrangle generates a projective subplane. In a stable plane, opposite sides of a quadrangle need not intersect, and the subplane generated by a quadrangle may even consist of just the quadrangle. The following result of Löwen [9: 1.33, 1.34] shows that there are always non-discrete subplanes that are finitely generated:

(3.4) LEMMA. Let $(u, v, e)$ be a triangle in a stable plane. Then there is a point $o$ and a neighbourhood $U$ of $u$ in $u v$ such that $(o, u, v, e)$ is a quadrangle and that for each point $b \in U \backslash\{u\}$, the (degenerated) quintangle $(o, u, v, e, b)$ generates a subplane $\mathbf{E}=(E, \mathcal{E})$ that lies dense in itself. If $\operatorname{dim} M \leq 4$, then $\operatorname{dim} E>0$.

(3.5) LEMMA. The line pencils of stable planes are locally homogeneous.

Proof. Let $X, Y$ be lines through a point $x$. Choose lines $G, H$ such that $g=G \wedge X$ and $h=H \wedge Y$ exist, and choose $z \in g h \backslash\{g, h\}$. Then $\pi=\pi_{x, G, z, H, x}: L \mapsto(((L \wedge G) z) \wedge H) x$ is defined on some neighbourhood $\mathcal{W}$ of $X$ in the line pencil, and $\pi$ is a homeomorphism of $\mathcal{W}$ onto the neighbourhood $\mathcal{W}^{\pi}$ of $Y$.

(3.6) DEFINITION. A full subplane $\mathbf{B}=(B, \mathcal{B})$ of a stable plane is called a Baer subplane, if $B$ is a closed subset of $M$ with $\operatorname{dim} B=\frac{1}{2} \operatorname{dim} M$.

For compact connected projective planes, this definition is equivalent with the usual one (i.e. that each line contains at least one point of $B$ ), see [20: 1.4], [21: 1.4].

(3.7) LEMMA. Let $\mathbf{M}=(M, \mathcal{M})$ be a stable plane. If there is a Baer subplane $\mathbf{B}=(B, \mathcal{B})$ such that $B$ is a (topological) manifold, then each line pencil of $\mathbf{M}$ (and hence each line and the point space $M$ ) is a manifold.

Proof. Choose a compact neighbourhood $V$ in $B$ and a point $x \in M \backslash B$. The mapping $\pi: V \rightarrow \mathcal{M}_{x}: b \mapsto b x$ is continuous and closed (since $V$ is compact). Since there is at most one line in $\mathcal{B}$ containing $x$, we may choose $V$ such that $\pi$ is injective. Then $V$ is homeomorphic with $\mathcal{W}=V^{\pi}$, hence $\operatorname{dim} \mathcal{W}=\operatorname{dim} \mathcal{M}_{x}$ and $\mathcal{W}$ contains a neighbourhood of some line in $\mathcal{M}_{x}$ by [11: Th. 11c)]. The assertion follows from local homogeneity (3.5).

(3.8) REMARK. If the point space of a stable plane has dimension $\leq 4$, then each line is a manifold [9: 1.13]. If $\operatorname{dim} M \leq 8$, the assumption that $B$ be a manifold is therefore superfluous. There is a conjecture that the point space is always a manifold (via the disjoint-disk property, see [11: Th. 9, Cor 10]), but the impact of the disjoint-disk property is dubious again, see [16]-[18].

If the lines of $\mathrm{M}$ are manifolds of dimension $l$, then each line pencil is homeomorphic with the sphere $S_{l}$ (see [9: 1.19]). In this case, useful information is obtained by an application of Alexander duality [5: VIII, 8.15]:

(3.9) LEMMA. Let $\mathrm{M}=(M, \mathcal{M})$ be a stable plane whose line pencils are homeomorphic with $S_{l}$, and let $(E, \mathcal{E})$ be a proper closed full subplane of $\mathbf{M}$ with $\operatorname{dim} E=2 e$. Then $\tilde{H}_{l-1-q}\left(\mathcal{M}_{x} \backslash \mathcal{E}_{x}\right) \cong \tilde{H}^{q}\left(\mathcal{E}_{x}\right)$ for each $q \in \mathbb{N}$ and each $x \in E$.

In particular, $\tilde{H}_{l-1-e}\left(\mathcal{M}_{x} \backslash \mathcal{E}_{x}\right) \neq 0$, and $\mathcal{M}_{x} \backslash \mathcal{E}_{x}$ is not homeomorphic with $\mathbf{R}^{l}$.

Applications of (3.9) will use the following 
(3.10) LEMMA. Let $\Lambda$ be a connected semi-planar group of a stable plane $\mathbf{M}=(M, \mathcal{M})$, write $F=(F, \mathcal{F})$ for the subplane induced on $F=\operatorname{Fix}(\Lambda)$, and let $x \in F$. If $\operatorname{dim} \Lambda=$ $\operatorname{dim} \mathcal{M}_{x}$ and $\Lambda_{L}=\mathbb{1}$ for some line $L$ through $x$, then the mapping $\mu: \Lambda \rightarrow \mathcal{M}_{x}: \lambda \mapsto L^{\lambda}$ is an open embedding. In particular, $\mathcal{M}_{x}$ is homeomorphic with a sphere, and $\Lambda$ is a Lie group. If $\Lambda_{L}=\mathbb{1}$ for each $L \in \mathcal{M}_{x} \backslash \mathcal{F}_{x}$, then $\Lambda$ contains an involution.

Proof. Assume that $\operatorname{dim} \Lambda=\operatorname{dim} \mathcal{M}_{x}$, and that $\Lambda_{L}=\mathbb{1}$ for some $L \in \mathcal{M}_{x}$. Then the mapping $\mu: \Lambda \rightarrow \mathcal{M}_{x}: \lambda \mapsto L^{\lambda}$ is a continuous injection. Restricting $\mu$ to some compact neighbourhood, one infers from [14: III.6] that $\operatorname{dim} \Lambda=\operatorname{dim} \Lambda^{\mu}$. Now [11: Th. 11c)] yields that $\Lambda^{\mu}=L^{\Lambda}$ is open in $\mathcal{M}_{x}$. According to [12: 6.3, p. 243] or [24], the group $\Lambda$ is a Lie group, and $\mathcal{M}_{x}$ is homeomorphic with a sphere. If $\Lambda_{L}=\mathbb{1}$ for each line $L \in \mathcal{M}_{x} \backslash \mathcal{F}_{x}$, we obtain that $L^{\Lambda}=\mathcal{M}_{x} \backslash \mathcal{F}_{x}$, since $\mathcal{M}_{x} \backslash \mathcal{F}_{x}$ is connected. Now (3.9) applies, yielding that $\Lambda$ has a non-trivial compact subgroup.

(3.11) LEMMA. Let $\Phi \leq$ Aut $(M)$ be a compact group fixing some point $x$. If there is a line $L \in \mathcal{M}_{x}$ such that $\operatorname{dim} L^{\Phi}=\operatorname{dim} \mathcal{M}_{x}$, then $\Phi$ acts transitively on $\mathcal{M}_{x}$.

Proof. Assume that $\operatorname{dim} L^{\Phi}=\operatorname{dim} \mathcal{M}_{x}$. According to [11: Th. 11c)], the compact orbit $L^{\Phi}$ is open in $\mathcal{M}_{x}$. Transitivity follows from the fact that $\mathcal{M}_{x}$ is connected [9: 1.14].

\section{RESTRICTIONS.}

In this section, let $M=(M, \mathcal{M})$ be a stable plane, and let $\Delta$ be a subgroup of Aut $(M)$. For subsets $X, Y$ of $M$ or $M$, let $[X, Y\rceil$ denote the set $\left\{\delta \in \Delta \mid X^{\delta} \subseteq Y\right\}$ and $\mathrm{Cp}(X)=\{C \subseteq X \mid C$ compact $\}, \mathrm{Op}(X)=\{U \subseteq X \mid U$ open $\}$.

We consider the following topologies on $\Delta$ :

a) The compact-open topology $\mathcal{T}$ derived from the action on $M$ is generated by the subbase $\{\lceil C, U\rceil \mid C \in \mathrm{Cp}(M), U \in \mathrm{Op}(M)\}$.

b) For each fixed point $o$ of $\Delta$, the topology $\mathcal{T}_{o}$ is generated by the subbase $\left\{\lceil C, U\rceil \mid C \in \mathrm{Cp}\left(\mathcal{M}_{o}\right), U \in \mathrm{Op}\left(\mathcal{M}_{o}\right)\right\}$

(i.e. the compact-open topology derived from the action on $\mathcal{M}_{0}$ ).

c) For any two fixed points $p, q$ of $\Delta$, the topology $\mathcal{T}_{p, q}$ is generated by the subbase $\left\{\lceil C, U\rceil \mid C \in \mathrm{Cp}\left(\mathcal{M}_{p} \times \mathcal{M}_{q}\right), U \in \mathrm{Op}\left(\mathcal{M}_{p} \times \mathcal{M}_{q}\right)\right\}$

(i.e. the compact-open topology derived from the action on $\mathcal{M}_{p} \times \mathcal{M}_{q}$ ).

d) For each fixed line $L$ of $\Delta$, the topology $\mathcal{T}_{M \backslash L}$ is generated by the subbase

$\{\lceil C, U\rceil \mid C \in \mathrm{Cp}(M \backslash L), U \in \mathrm{Op}(M \backslash L)\}$

(i.e. the compact-open topology derived from the action on $M \backslash L$ ).

Our aim is to show that the topologies $\mathcal{T}_{0}$ and $\mathcal{T}$ coincide in the case where $\Delta$ is a semiplanar group (this is analogous to the result of T. Grundhöfer [7: p.297 (in the proof of the corollary)] for the case of compact (possibly disconnected) projective planes). Since each of the considered topologies makes $\Delta$ a topological semi-group, it suffices to consider the neighbourhoods of 1 .

(4.1) REMARK. The actions of $\Delta$ on $\mathcal{M}_{o}, \mathcal{M}_{p} \times \mathcal{M}_{q}$ and $M \backslash L$ are continuous with respect to $\mathcal{T}$, and we infer that $\mathcal{T}_{o}, \mathcal{T}_{p, q}$ and $\mathcal{T}_{M \backslash L}$ are contained in $\mathcal{T}$ (cf. [13: $\S 7-6$, Ex. 5, p. 289]). 
(4.2) LEMMA. If $\triangle$ fixes two points $p, q$, then $\mathcal{T}_{p} \subseteq \mathcal{T}_{p, q}$.

Proof. The set $\left\{[\mathcal{C} \times \mathcal{D}, \mathcal{W}\rceil \mid \mathcal{C} \in \mathrm{Cp}\left(\mathcal{M}_{p}\right), \mathcal{D} \in \mathrm{Cp}\left(\mathcal{M}_{q}\right), \mathcal{W} \in \mathrm{Op}\left(\mathcal{M}_{p} \times \mathcal{M}_{q}\right)\right\}$ forms a subbase for $\mathcal{T}_{p, q}$ (cf. [6: Ex.1, p. 264]). The sets $\mathcal{M}_{p}$ and $\mathcal{M}_{q}$ are $\Delta$-equivariantly embedded in $\mathcal{M}_{p} \times \mathcal{M}_{q}$ via $\iota_{p}: L \mapsto(L, p q)$ and $\iota_{q}: L \mapsto(p q, L)$, respectively. This yields that $\mathcal{T}_{p} \subseteq \mathcal{T}_{p, q}$

(4.3) LEMMA. The topology $\mathcal{T}_{p, q}$ is generated by the subbase

$$
\left\{\lceil\mathcal{C} \times \mathcal{D}, \mathcal{U} \times \mathcal{V}\rceil \mid \mathcal{C} \in \mathrm{Cp}\left(\mathcal{M}_{p}\right), \mathcal{D} \in \mathrm{Cp}\left(\mathcal{M}_{q}\right), \mathcal{U} \in \mathrm{Op}\left(\mathcal{M}_{p}\right), \mathcal{V} \in \mathrm{Op}\left(\mathcal{M}_{q}\right)\right\}
$$

In particular, $\mathcal{T}_{p} \cup \mathcal{T}_{q}$ is a subbase for $\mathcal{T}_{p, q}$.

Proof. Assume that $\mathbb{1} \in[\mathcal{C} \times \mathcal{D}, \mathcal{W}\rceil$. There are open neighbourhoods $\mathcal{U}, \mathcal{V}$ of $\mathcal{C}, \mathcal{D}$ in $\mathcal{M}_{p}, \mathcal{M}_{q}$, respectively, such that $\mathcal{U} \times \mathcal{V}$ is contained in $\mathcal{W}$. Consequently, the neighbourhood $[\mathcal{C} \times \mathcal{D}, \mathcal{U} \times \mathcal{V}\rceil$ is contained in $[\mathcal{C} \times \mathcal{D}, \mathcal{W}\rceil$. Since $\mathcal{U} \times \mathcal{V}$ is open in $\mathcal{M}_{p} \times \mathcal{M}_{q}$, we obtain the first part of the assertion. The equality $\lceil\mathcal{C} \times \mathcal{D}, \mathcal{U} \times \mathcal{V}\rceil=[\mathcal{C}, \mathcal{U}\rceil \cap[\mathcal{D}, \mathcal{V}\rceil$ yields the second part.

(4.4) LEMMA. If $p, q$ are two fixed points of $\Delta$ and $L=p q$, then $\mathcal{T}_{p, q} \supseteq \mathcal{T}_{M} \backslash L$.

Proof. Let $C \in \operatorname{Cp}(M \backslash L)$ and $U \in \mathrm{Op}(M \backslash L)$ such that $\mathbb{1} \in[C, U]$ (i.e. $C \subseteq U$ ). For each point $c \in C$, there are compact neighbourhoods $\mathcal{U}_{c}, \mathcal{V}_{c}$ of $p c, q c$ in $\mathcal{M}_{p}, \mathcal{M}_{q}$, respectively, such that $\mathcal{W}_{c}=\mathcal{U}_{c} \times \mathcal{V}_{c}$ consists of pairs of intersecting lines and that $W_{c}=$ $\left\{G \wedge H \mid(G, H) \in \mathcal{W}_{\mathrm{c}}\right\}$ is contained in $U$. The mapping

$$
\sigma: m \mapsto(p m, q m): M \backslash L \rightarrow \mathcal{M}_{p} \times \mathcal{M}_{q}
$$

is a continuous open injection, hence $W_{c}$ is a compact neighbourhood of $c$ in $M \backslash L$. Since $C$ is compact, there is a finite number of points $c_{3}, \ldots, c_{n}$ such that $C \subseteq \bigcup_{i=1}^{n} W_{c_{i}}$. Consequently, $\lceil C, U\rceil \supseteq \bigcap_{i=1}^{n}\left\lceil W_{c_{i}}, U\right\rceil \ni \mathbb{1}$. Now $\left\lceil W_{c}, U\right\rceil=\left\lceil\mathcal{W}_{c}, U^{\sigma}\right\rceil$, and $\bigcap_{i=1}^{n}\left\lceil W_{c_{i}}, U\right\rceil$ belongs to $\mathcal{T}_{p, q}$.

(4.5) LEMMA. If $\Delta$ is semi-planar, then $\mathcal{T}_{o}=\mathcal{T}_{p, q}$ for each choice $o, p, q \in \operatorname{Fix}(\Delta)$ such that $p \neq q$.

Proof. Assume first that $o=p$. Let $[\mathcal{C} \times \mathcal{D}, \mathcal{U} \times \mathcal{V}\rceil$ be a $\mathcal{T}_{p, q}$-neighbourhood of $\mathbb{1}$ (cf. (4.3)). In particular, the set $\mathcal{D}$ is contained in $\mathcal{V}$. We may assume that $\mathcal{D} \neq \mathcal{M}_{q}$ (otherwise $\left.\lceil\mathcal{C} \times \mathcal{D}, \mathcal{U} \times \mathcal{V}\rceil=\lceil\mathcal{C}, \mathcal{U}\rceil \in \mathcal{T}_{p}\right)$ and even that the closure of $\mathcal{V}$ is a proper subset of $\mathcal{M}_{q}$ (since $\mathcal{M}_{q}$ is a regular space). Now there is a line $H$ that is fixed by $\Delta$, does not pass through $p$ or $q$ and meets each line in $\mathcal{V}$. On the open set $\mathcal{W}=\left\{L \in \mathcal{M}_{q} \mid L\right.$ meets $\left.H\right\}$ we define the local projectivity $\mu: L \mapsto(L \wedge H) p$. Now $\mu$ commutes with $\Delta$ (since $p, q$ and $H$ are fixed by $\Delta$ ) and is a homeomorphism of $\mathcal{W}$ onto the open subset $\mathcal{W}^{\mu}$ of $\mathcal{M}_{p}$. This yields that $[\mathcal{D}, \mathcal{V}\rceil=\left\lceil\mathcal{D}^{\mu}, \mathcal{V}^{\mu}\right\rceil \in \mathcal{T}_{p}$, and $\lceil\mathcal{C} \times \mathcal{D}, \mathcal{U} \times \mathcal{V}\rceil \in \mathcal{T}_{p}$. Therefore $\mathcal{T}_{p, q} \subseteq \mathcal{T}_{p}$ and the assertion follows from (4.2) for the case where $o=p$. If $o \neq p$, we conclude that $\mathcal{T}_{o}=\mathcal{T}_{o, p}=\mathcal{T}_{p}=\mathcal{T}_{p, q}=\mathcal{T}_{q}$

(4.6) PROPOSITION. If $\Delta$ is semi-planar, then $\mathcal{T}=\mathcal{T}_{o}$ for each fixed point of $\Delta$.

Proof. Let $[C, U]$ be an element of $\mathcal{T}$. For each point $c \in C$, there are two fixed points $p_{c}, q_{c}$ of $\Delta$ and a compact neighbourhood $D_{c}$ of $c$ such that the line $L_{c}=p_{c} q_{c}$ and $D_{c}$ are disjoint. Since $C$ is compact, there is a finite number of points $c_{1}, \ldots, c_{n}$ such that $C \subseteq \bigcup_{i=1}^{n} D_{c_{i}}$. Hence $\lceil C, U\rceil \supseteq \bigcap_{i=1}^{n}\left\lceil W_{c_{i}}, U\right\rceil$. But $\left\lceil D_{c_{i}}, U\right\rceil \in \mathcal{T}_{M} \backslash L_{c_{i}} \subseteq \mathcal{T}_{p_{c_{i}}, q_{c_{i}}}=\mathcal{T}_{o}$ (according to (4.4) and (4.5)), and we have shown that $\mathcal{T} \subseteq \mathcal{T}_{0}$. Since $\mathcal{T}_{0} \subseteq \mathcal{T}$ (cf. (4.1)), the assertion follows. 


\section{THE LOW DIMENSIONS.}

In the case of stable planes of dimension at most 4, subplanes and (semi-)planar groups are well understood. According to [9: 1.34], there are no non-discrete closed full subplanes of dimension 0 (i.e. each semi-planar group is actually planar), and the possible planar groups are known explicitly:

(5.1) THEOREM. Let $\Lambda$ be a semi-planar group of a stable plane $M=(M, M)$.

a) If $\operatorname{dim} M=2$, then $\Lambda$ is trivial.

b) If $\operatorname{dim} M=4$, then $\Lambda$ consists of at most two elements.

Proof. By the maximum property (3.3) and (3.4), we have that $\operatorname{dim} \operatorname{Fix}(\Lambda)=2$ and $\operatorname{dim} M=4$ whenever $\Lambda$ is not trivial. In this case, each non-trivial element of $\Lambda$ is an involution by [10: 1.6] and reverses orientation on the line pencil in any fixed point. Consequently, the product of two non-trivial elements is trivial, and $\Lambda \cong \mathbb{Z}_{2}$.

(5.2) COROLLARY. Let $\Lambda$ be a connected semi-planar group of a stable plane $\mathbf{M}=$ $(M, \mathcal{M})$, and $c \in M \backslash F i x(\Lambda)$. Then the subplane generated by $\operatorname{Fix}(\Lambda) \cup c^{\Lambda}$ has dimension 8 at least.

Warning. There may be non-continuous planar collineations (e.g. the collineations that are induced on the projective plane over the field $C$ of complex numbers by non-continuous automorphisms of $\mathrm{C}$ ). These need not be involutions, nor do they always commute.

\section{COMPACTNESS.}

For compact connected projective planes, it seems reasonable to conjecture that each semi-planar group (even each stabilizer of a quadrangle) is compact. No example of a noncompact semi-planar group is known, but there is still no general proof of compactness. Several rather complicated compactness criteria have been stated for the projective case (e.g. [21: 2.2-2.4]). In the case of stable planes, we can prove the following:

(6.1) PROPOSITION. Let $M$ be a stable plane. Any closed subgroup of Aut (M) that acts trivially on a Baer subplane $\mathbf{B}=(B, \mathcal{B})$ is compact.

Proof. Assume that a non-compact group $\Lambda$ satisfies the hypotheses of the proposition. Let $(o, u, v)$ be a triangle in $\mathbf{B}$. According to (4.6), the group $\Lambda$ is homeomorphic to its restriction $\left.\Lambda\right|_{\mathcal{M}_{o}}$. By the Arzela-Ascoli theorem [13: 7-6.1, p. 290], there are a sequence of lines $L_{n} \in \mathcal{M}_{u}$ and a sequence of automorphisms $\lambda_{n} \in \Lambda$ such that $\lim L_{n}=$ ou but ou is no cluster point of the sequence $L_{n}^{\lambda_{n}}$. Choose $J \in \mathcal{M}_{v} \backslash \mathcal{B}_{v}$ such that $J \wedge o u$ exists. There are compact neighbourhoods $O$ of $o$ in $o v$ and $U$ of $u$ in $u v$ such that $J$ intersects each line $t s$, where $t \in O, s \in U$. Put $D=(B \cap U) \times(B \cap O)$. Since $B$ is closed in $M$, the set $D$ is compact, and $\operatorname{dim} D=\operatorname{dim} B$. Now the mapping $\mu: D \rightarrow \mathcal{M}_{u}:(s, t) \mapsto(s t \wedge J) u$ is a closed continuous injection (recall that $J \notin \mathcal{B}$ ). From [11: Th. 11c)] we obtain that $D^{\mu}$ is a neighbourhood in $\mathcal{M}_{u}$, and we may assume that $\left\{L_{n} \mid n \in \mathbb{N}\right\} \subseteq D^{\mu}$. For each pair $\left(s_{n}, t_{n}\right) \in D$ with $L_{n}=\left(s_{n}, t_{n}\right)^{\mu}$ we have that $\lim \left(s_{n}, t_{n}\right)=(u, o)$. Since ou is not a cluster point of $L_{n}^{\lambda_{n}}$, the sequence $\left(J \wedge L_{n}\right)^{\lambda_{n}}=\left(s_{n} t_{n} \wedge L_{n}\right)^{\lambda_{n}}=s_{n} t_{n} \wedge L_{n}^{\lambda_{n}}$ converges to $u$. Hence $\lim J^{\lambda_{n}}=w v$.

According to (3.5), there are lines $G \in \mathcal{B}_{u}, H \in \mathcal{B}_{o}$ and a point $z \in o u \cap B$ such that $\pi=$ $\pi_{v, G, z, H, v}$ is a homeomorphism of some neighbourhood $\mathcal{W}$ of $u v$ in $\mathcal{M}_{v}$ onto a neighbour- 
hood of $o v$ in $\mathcal{M}_{v}$. We may choose $J \in \mathcal{W}$ and put $K=J^{\pi}$. Since $v, G, z, H$ belong to $B$, they are fixed by $\Lambda$, and $\Lambda$ centralizes $\pi$. Hence $\lim K^{\lambda_{n}}=(u v)^{\pi}=o v$. For $d \in B \cap u v$ near $u$, the line $(d, o)^{\mu}$ and the point $(d, o)^{\mu} \wedge K$ exist, and there are points $p \in u v \cap B$ and $q \in$ ov $\cap B$ such that $(d, o)^{\mu}=(p q \wedge K) u$. Now from $\left((d, o)^{\mu}\right)^{\lambda_{n}}=\left(d o \wedge J^{\lambda_{n}}\right) u$ we have that $\lim \left((d, o)^{\mu}\right)^{\lambda_{n}}=d u=u v$. On the other hand, we obtain from $\left((d, o)^{\mu}\right)^{\lambda_{n}}=\left(p q \wedge K^{\lambda_{n}}\right) u$ that $\lim \left((d, o)^{\mu}\right)^{\lambda_{n}}=q u$. This contradiction proves the proposition.

Typically, Baer subplanes occur with planar involutions (see [23: 3.7] for a proof):

(6.2) LEMMA. Let $\alpha$ be an involutory automorphism of a stable plane $\mathbf{M}=(M, \mathcal{M})$. If Fix $(\alpha)$ contains a quadrangle, then the geometry $\mathrm{F}_{\alpha}=\left(\operatorname{Fix}(\alpha), \mathcal{F}_{\alpha}\right)$ induced on $\mathrm{Fix}(\alpha)$ is a Baer subplane.

In the case of eight-dimensional planes, we obtain

(6.3) PROPOSITION. Let $\mathrm{M}=(M, \mathcal{M})$ be a stable plane with $\operatorname{dim} M=8$. If a connected subgroup $\Lambda$ of Aut (M) leaves invariant a Baer subplane $\mathbf{B}=(B, \mathcal{B})$ and induces a semiplanar group on $\mathrm{B}$, then $\Lambda$ acts trivially on $\mathrm{B}$, and $\Lambda$ is a compact abelian group with $\operatorname{dim} \Lambda \leq 1$.

Proof. According to (5.1), $\Lambda$ acts trivially on $\mathrm{B}$. Let $(o, u, v)$ be a triangle in $B$. The restriction of $\Lambda$ to $\mathcal{M}_{0}$ is effective, and the lines that are fixed by $\Lambda$ form a subset homeomorphic with $S_{2}$ in $\mathcal{M}_{0}$. According to Richardson's classification of actions on the 4sphere [19], the group $\Lambda$ has no orbit of dimension $\geq 2$ in $\mathcal{M}_{o}$. For $x \in u v \backslash B$, we infer that $\operatorname{dim} x^{\Lambda}=\operatorname{dim}(x o)^{\Lambda} \leq 1$. Since $\langle B \cup\{x\}\rangle=\mathbf{M}$, this yields that $\Lambda_{x}=\mathbb{1}$ and $\operatorname{dim} \Lambda \leq 1$.

(6.4) COROLLARY. Let $\Lambda$ be a semi-planar group of an eight-dimensional stable plane, and let $\alpha$ be an involution contained in $\Lambda$. Then the identity component of the centralizer $\mathrm{C}_{\Lambda}(\alpha)$ is compact, and $\operatorname{dim} \mathrm{C}_{\Lambda}(\alpha) \leq 1$.

(6.5) REMARK. Let us state one of the typical applications of results on semi-planar groups, namely that the dimension bound in (6.3) restricts the possibilities for semi-simple groups of automorphisms of eight-dimensional stable planes: If such a group centralizes a planar involution $\alpha$, then it acts almost effectively on $F_{\alpha}$, inducing one of the almost simple Lie groups listed by Löwen [10]:

$$
\begin{gathered}
\mathrm{PSL}_{3} \mathrm{C}, \quad \mathrm{PSU}_{3} \mathrm{C}, \quad \mathrm{PSU}_{3} \mathrm{C}(1) \\
\mathrm{PSL}_{3} \mathrm{R}, \quad \mathrm{SL}_{2} \mathrm{C}, \quad \mathrm{PSL}_{2} \mathrm{C} \cong \Omega_{4} \mathrm{R}(1) \cong \mathrm{SO}_{3} \mathrm{C} \\
\mathrm{SU}_{2} \mathrm{C}, \quad \mathrm{SO}_{3} \mathrm{R}, \quad \mathrm{SL} \mathrm{L}_{2} \mathrm{R}, \quad \mathrm{PSL}_{2} \mathrm{R} \cong \Omega_{3} \mathrm{R}(1) \text {, or a covering of } \mathrm{PSL}_{2} \mathrm{R} .
\end{gathered}
$$

(6.6) LEMMA. Let $\Lambda$ be a connected semi-planar group of $\mathrm{M}$, and let $\mathbf{D}=(D, \mathcal{D})$ and $\mathrm{E}=(E, \mathcal{E})$ be two $\Lambda$-invariant Baer subplanes such that $D \cap E$ carries a Baer subplane of $\mathrm{D}$ and $\operatorname{Fix}(\Lambda) \subseteq D \cap E$. Then $\Lambda$ is abelian, and $\operatorname{dim} \Lambda \leq 2$.

Proof. We may assume that $\operatorname{dim} M=16$. Since $\operatorname{dim} D \cap E \leq 4$, the set $F i x(\Lambda)$ has dimension 2 or 4 by (3.4). Thus $F=\langle F i x(\Lambda)\rangle$ equals $G=\langle D \cap E\rangle$, or $F$ is a Baer subplane of $G$. Now (6.3) yields that $\Lambda$ induces an abelian group of dimension $\leq 1$ on $D$ and $E$, respectively. On the other hand, the kernel of the restriction to $D$ acts effectively on $E$, and vice versa. Therefore $\Lambda$ is the direct product of these kernels, and the assertion follows.

In a way, the number of commuting involutions restrains the size and structure of compact (Lie) groups. Hence the following will be useful: 
(6.7) LEMMA. Let $\Lambda$ be a semi-planar group, and let $\alpha, \beta \in \Lambda$ be two commuting involutions. Then $\operatorname{Fix}(\alpha) \neq \operatorname{Fix}(\beta)$, and the identity component of $\mathrm{C}_{\Lambda}(\alpha, \beta)$ is an abelian group of dimension $\leq 2$.

Proof. By (5.1), we need only consider 8- and 16-dimensional planes. But here a theorem by $P$. Smith [22] applies to the action of $\langle\alpha, \beta\rangle$ on the line pencil in some fixed point, namely: for $l>2$, there is no action of $\mathbb{Z}_{2} \times \mathbb{Z}_{2}$ on a homotopy $2 l$-sphere such that the set of fixed points forms an $l$-sphere. Now let $\Psi$ be the centralizer $C_{\Lambda}(\alpha, \beta)$. Then the fixed planes $F_{\alpha}$ and $F_{\beta}$ are $\Psi$-invariant Baer subplanes, and (6.6) applies.

(6.8) COROLLARY. If a compact connected non-trivial Lie group $\Lambda \leq A u t(M)$ acts trivially on some Baer subplane, then $\Lambda$ is isomorphic with $\mathrm{SO}_{2} \mathrm{R}$ or $\mathrm{SU}_{2} \mathrm{C}$.

Applying this result inductively, starting from (5.1), we obtain:

(6.9) COROLLARY. Let $\Theta$ be a compact Lie group of rank $r>1$ acting effectively as a semi-planar group of a stable plane $\mathrm{M}=(M, \mathcal{M})$. Then $\operatorname{dim} M \geq 2^{r+2}$.

The structure theorem on compact connected groups [2: App. I, no. 3, Prop. 2] provides the following information: For each compact connected group $\Phi$ there is an epimorphism $\pi: C \times S \rightarrow \Phi$, where $C$ is a compact connected abelian group, and $S$ is a direct product of (possibly infinitely many) compact almost simple Lie groups. Note that the commutator subgroup of $\Phi$ equals $S^{\pi}$, and that $C^{\pi}$ is the identity component of the center of $\Phi$. In the sequel, we will use this to derive information about the size and the structure of compact connected semi-planar groups.

(6.10) LEMMA. Let $\Phi$ be a compact abelian semi-planar group of $\mathrm{M}=(M, \mathcal{M})$. Then $\operatorname{dim} \Phi<\frac{1}{2} \operatorname{dim} M$.

Proof. Choose a triangle $(o, u, v)$ in $\operatorname{Fix}(\Phi)$. If there is a point $c \in u v$ such that the set Fix $(\Phi) \cup c^{\Phi}$ generates $M$, then $\Phi_{o c}=\Phi_{c}=\mathbb{1}$ and $\operatorname{dim} \Phi<\frac{1}{2} \operatorname{dim} M$ by (3.11). If, on the other hand, the set $\operatorname{Fix}(\Phi) \cup c^{\Phi}$ generates a Baer subplane for each $c \in u v \backslash$ Fix $(\Phi)$, then $\operatorname{dim} c^{\Phi}<\frac{1}{4} \operatorname{dim} M$. Choosing $d \in u v$ outside the subplane generated by $\operatorname{Fix}(\Phi) \cup c^{\Phi}$, one infers that $\Phi_{c, d}=\mathbb{1}$ and $\operatorname{dim} \Phi<\frac{1}{2} \operatorname{dim} M$.

(6.11) LEMMA. Let $\Phi$ be a compact connected semi-planar group of $\mathrm{M}=(M, \mathcal{M})$. If the commutator subgroup $\Phi^{\prime}$ has rank 2 , then $\Phi=\Phi^{\prime}$, and $\Phi$ is isomorphic with the compact exceptional Lie group of type $\mathrm{G}_{2}(-14)$, the group $\mathrm{SU}_{3} \mathrm{C}$, or $\mathrm{SO}_{4} \mathrm{R}$.

Proof. If $\Phi^{\prime}$ has rank 2 , then there is a subgroup isomorphic with $\mathrm{SO}_{2} \mathbf{R} \times \mathrm{SO}_{2} \mathbf{R}$. Since this is an abelian group of dimension 2 containing two commuting involutions, the identity component of the center of $\Phi$ is trivial by (6.7), and $\Phi=\Phi^{\prime}$.

i) Each semi-simple compact group of rank 2 is isomorphic with the compact exceptional Lie group of type $\mathrm{G}_{2}(-14)$ or locally isomorphic with one of the groups $\mathrm{SO}_{5} \mathrm{R}, \mathrm{SU}_{3} \mathrm{C}$ or $\mathrm{SO}_{4} \mathrm{R}$. The two-fold covering group $\mathrm{Spin}_{4}$ of $\mathrm{SO}_{4} \mathrm{R}$ has three central involutions. Each of the groups $\mathrm{SO}_{5} \mathrm{R}, \mathrm{SO}_{3} \mathrm{R} \times \mathrm{SU}_{2} \mathrm{C}$ and $\mathrm{SO}_{3} \mathrm{R} \times \mathrm{SO}_{3} \mathrm{R}$ contains a pair of commuting involutions whose centralizer is more than 2-dimensional. By (6.7), only the case that $\Phi \cong \mathrm{PSU}_{3} \mathrm{C}$ remains to be excluded.

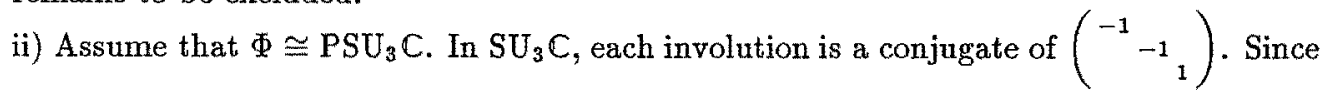
the center of $\mathrm{SU}_{3} \mathrm{C}$ has order 3 , the canonical epimorphism onto $\Phi$ is a one-to-one mapping of the involutions. Hence the involutions of $\Phi$ form a single conjugacy class. Let $\alpha$ be an involution of $\Phi$, and $\Sigma T$ its centralizer in $\Phi$, where $\Sigma \cong \mathrm{SU}_{2} \mathrm{C}$ is the commutator subgroup 
and $\mathrm{T} \cong \mathrm{SO}_{2} \mathrm{R}$ is the center of $\mathrm{C}_{\Phi}(\sigma)$. Since $\Sigma \mathrm{T}$ is maximal in $\Phi$, the global stabilizer of $F_{\alpha}$ equals $\Sigma \mathrm{T} . \mathrm{By}(6.9)$ and (6.7), the group $\Sigma \mathrm{T}$ induces a group isomorphic with $\mathrm{SO}_{3} \mathrm{R}$ or $\mathrm{SO}_{2} \mathrm{~F}$ on $\mathrm{F}_{\alpha}$. In both cases, an involution is induced on $\mathrm{F}_{\alpha}$. Using (3.4), we infer that $\Phi$ is planar. For each point $x \in \operatorname{Fix}(\alpha) \backslash \operatorname{Fix}(\Phi)$, we obtain that $\operatorname{Fix}(\Phi) \cup\{x\}$ generates $F_{\alpha}$, and $(\Sigma T)_{x}$ equals the kernel of the action on $F_{\alpha}$.

iii) We determine the stabilizers of lines through a point $o \in \operatorname{Fix}(\Phi)$ : Let $L \in \mathcal{M}_{0}$ be a line that is moved by $\Phi$. Then there is a line $H$ that is fixed by $\Phi$ such that $H$ meets $L$ in a point $x \neq o$. Since $H$ is fixed by $\Phi$, the stabilizer $\Phi_{x}=\Phi_{L}$ has dimension $\geq 1$ by (3.11). Consequently, we may assume that $\alpha \in \Phi_{x}$, and $x \in \mathrm{F}_{\alpha}$. Combining this with ii), we infer that each stabilizer of a line through $o$ that is moved by $\Phi$ is a conjugate of $\Phi_{L}=\Phi_{x}$. Now Bredon's results [3], [4] yield that $L^{\Phi}$ is a sphere. The exact homotopy sequence shows that $\pi_{1}(\Phi) \cong \pi_{1}\left(\Phi_{L}\right)=0$, a contradiction.

(6.12) LEMMA. Let $\Phi$ be a compact connected semi-planar group of $\mathbf{M}=(M, \mathcal{M})$. If $\operatorname{dim} Z(\Phi) \neq 0$, then $\operatorname{dim} \Phi^{\prime} \leq 3$, and $\operatorname{dim} \Phi<\frac{1}{2} \operatorname{dim} M$.

Proof. By (6.10) and (6.11), we may assume that $\Phi^{\prime}$ has rank 1. Consequently, the group $\Phi^{\prime}$ is isomorphic with $\mathrm{SO}_{3} \mathrm{R}$ or its covering $\mathrm{SU}_{2} \mathrm{C}$. In the first case, there are two commuting involutions in $\Phi^{\prime}$. From (6.7) we infer that $\operatorname{dim} Z(\Phi)=1$, and $\operatorname{dim} \Phi \leq 4$. If $\Phi \cong \mathrm{SU}_{2} \mathrm{C}$, then the involution $\sigma \in \Phi^{\prime}$ centralizes $\Phi$. Let $c$ be a point on a line that is fixed by $\Phi$ such that $c$ is moved by both $\sigma$ and the identity component of $\mathrm{Z}(\Phi)$. If $\left\langle\operatorname{Fix}(\Phi) \cup c^{Z(\Phi)}\right\rangle=M$, then $\Phi_{c}$ is trivial and $\operatorname{dim} \Phi<\frac{1}{2} \operatorname{dim} M$ by (3.11). If, on the other hand, the set $\mathrm{Fix}(\Phi) \cup c^{\mathrm{Z}(\Phi)}$ generates a Baer subplane $\mathrm{B}$ of $\mathrm{M}$, then $\mathrm{Z}(\Phi)$ leaves invariant both $B$ and $F_{\sigma}$. According to $(6.6), \operatorname{dim} Z(\Phi) \leq 2$, and $\operatorname{dim} \Phi \leq 5$.

(6.13) THEOREM. Let $\Phi$ be a non-trivial compact connected semi-planar group of a stable plane $\mathbf{M}=(M, \mathcal{M})$.

a) If $\operatorname{dim} M=8$, then $\Phi \cong \mathrm{SO}_{3} \mathrm{R}, \Phi \cong \mathrm{SO}_{2} \mathrm{R}$ or $\Phi$ is an abelian non-Lie group. In any case, $\operatorname{dim} \Phi \leq 3$.

b) If $\operatorname{dim} M=16$, then $\Phi$ is isomorphic with one of the groups $\mathrm{G}_{2}(-14), \mathrm{SU}_{3} \mathrm{C}, \mathrm{SO}_{4} \mathbb{R}, \mathrm{U}_{2} \mathbb{C}, \mathrm{SU}_{2} \mathbb{C}, \mathrm{SO}_{3} \mathbb{R}, \mathrm{SO}_{2} \mathrm{R} \times \mathrm{SO}_{2} \mathrm{R}, \mathrm{SO}_{2} \mathrm{R}$, or $\Phi$ is a non-Lie group of dimension $\leq 7$.

Proof. The assertions for the case where $\operatorname{dim} M=8$ follow from (6.4), (6.9) and (6.10). If $\operatorname{dim} M=16$, combine (6.11), (6.12) and (6.7).

(6.14) Remark. Note that the Lie groups listed in (6.13) are subgroups of the automorphism groups $\mathrm{SO}_{3} R$ of Hamilton's quaternions and $\mathrm{G}_{2}(-14)$ of Cayley's octonions, respectively.

\section{SEMI-PLANAR GROUPS OF EIGHT-DIMENSIONAL PLANES.}

In this section, let $M=(M, \mathcal{M})$ be an eight-dimensional stable plane (unless stated otherwise), and let $\Lambda$ be a connected semi-planar group of $M$, with $F=(F, \mathcal{F})$, where $F=$ Fix $(\Lambda)$. Choose a triangle $(o, u, v)$ in $F$. We are going to derive a bound for the dimension of $\Lambda$. Unfortunately, this bound exceeds the bounds in the "classical situation"; i.e. our bound is greater than the dimension of the stabilizer of a quadrangle in the projective plane over Hamilton's quaternions. No example for such a semi-planar group is known, however. On the other hand, the bound obtained for planar groups is sharp. 
(7.1) LEMMA. Let $\mathbb{E}=(E, \mathcal{E})$ be a proper closed full subplane of positive dimension with $F \subseteq E$. Then $\operatorname{dim} c^{\Lambda} \leq 2$ for each point $c \in E \cap o u$.

Proof. Since the assertion is trivial if $E=F$, we may assume that $\operatorname{dim} F=2$ and $\operatorname{dim} E=4$. For $c \in(E \cap o u) \backslash F$, we have that $\mathbb{E}=\langle F \cup\{c\}\rangle$, and $\Lambda_{c}$ acts trivially on $\mathrm{E}$. By (6.3), the identity component of the global stabilizer $\Lambda_{E}$ acts trivially on $E$ and therefore equals the identity component of $\Lambda_{c}$. In particular, $\Lambda_{E}$ and $\Lambda_{c}$ have the same dimension. Put $\eta:(E \cap o u) \times \Lambda \rightarrow o u:(x, \lambda) \mapsto x^{\lambda^{-1}}$. Restricting $\eta$ to some compact neighbourhood, we obtain from [14: III.6] that there exists $b \in o u$ such that $\operatorname{dim} b^{\eta} \geq \operatorname{dim} \Lambda+2-4=$ $\operatorname{dim} \Lambda-2$, where $b^{\eta^{-}}=\left\{(x, \lambda) \mid x^{\lambda^{-1}}=b\right\}$. Now there is $\delta \in \Lambda$ such that $b^{\delta} \in E \cap o u$, and $\mathbb{E}=\left\langle F \cup\left\{b^{\delta}\right\}\right\rangle$. We may assume that $\delta=\mathbb{1}$. From

$$
b^{\eta^{-}}=\left\{(x, \lambda) \mid E \ni x=b^{\lambda}\right\}=\left\{\left(b^{\lambda}, \lambda\right) \mid b^{\lambda} \in E\right\}=\left\{\left(b^{\lambda}, \lambda\right) \mid \lambda \in \Lambda_{E}\right\}
$$

we infer that $\Lambda_{E}$ is locally homeomorphic with $b^{\eta^{-}}$.

This yields that $\operatorname{dim} \Lambda_{c}=\operatorname{dim} \Lambda_{E} \geq \operatorname{dim} \Lambda-2$, hence $\operatorname{dim} c^{\Lambda} \leq 2$.

(7.2) COROLLARY. If $F$ is contained in some Baer subplane $B$ of $\mathrm{M}$, then $\operatorname{dim} \Lambda \leq 3$.

Proof. According to [9: 1.34], we have that $\operatorname{dim} F \geq 2$, and $\langle F \cup\{c\}\rangle=\mathbb{B}$ for each $c \in B \backslash F$. Now $\operatorname{dim} \Lambda_{c} \leq 1$ by (6.3), and from (7.1) it follows that $\operatorname{dim} \Lambda \leq 3$.

(7.3) PROPOSITION. If $\Lambda$ is a planar group of an eight-dimensional plane, then $\operatorname{dim} \Lambda \leq 3$.

Proof. By (7.2), we need only consider the case where $F$ is a maximal subplane and $\operatorname{dim} F=2$. For each line $L \in \mathcal{M}_{0} \backslash \mathcal{F}_{0}$, there is a line $H \in \mathcal{F}$ that meets $L$ such that $L \wedge H \neq o$. Since $\langle F \cup\{L \wedge H\}\rangle=\mathrm{M}$, we obtain that $\Lambda_{L}=\mathbb{1}$ and $\operatorname{dim} \Lambda \leq 4$. If $\operatorname{dim} \Lambda=4$, we infer from (3.10) that $\Lambda$ is a lie group containing an involution. This contradicts (7.2).

(7.4) LEMMA. Let $\Phi$ be a connected, non-trivial subgroup of $\Lambda$. Then $\operatorname{dim} \mathrm{C}_{\Lambda}(\Phi) \leq 3$.

Proof. Let $E=(\operatorname{Fix}(\Phi), \mathcal{E})$, and let $\Psi$ be the centralizer of $\Phi$ in $\Lambda$. For each line $L \in \mathcal{M}_{o} \backslash \mathcal{F}_{o}$, there is a line $H \in \mathcal{F}$ that meets $L$ such that $L \wedge H \neq o$. By (5.2), we have that $\left\langle F \cup x^{\Phi}\right\rangle=\mathrm{M}$. Consequently, the stabilizer $\Psi_{L}$ is trivial, and $\operatorname{dim} \Psi \leq 4$. If $\operatorname{dim} \Psi=4$, then (3.10) yields that $\Psi$ is a Lie group containing an involution $\alpha$. But now $F \subseteq F_{\alpha}$ and $\operatorname{dim} \Lambda \leq 3$, a contradiction.

(7.5) PROPOSITION. If $\Lambda$ is semi-simple, then it is even almost simple, and $\operatorname{dim} \Lambda \leq 3$.

Proof. If $\Lambda$ is semi-simple, but not almost simple, then choosing a one-parameter subgroup $P$ in one of the almost simple factors one obtains that $\operatorname{dim} C_{\Lambda}(P) \geq 4$ in contradiction to (7.4). Each almost simple group of dimension greater than 3 contains a compact semisimple group and thus an involution. So (7.2) proves the assertion.

(7.6) THEOREM. (Rigidity properties of eight-dimensional planes)

Let $\Lambda$ be a non-trivial semi-planar group of an eight-dimensional stable plane. Then $\operatorname{dim} \Lambda+\operatorname{dim} \operatorname{Fix}(\Lambda) \leq 5$.

Proof. According to (6.3) and (7.5), we may assume that $\Lambda$ is not semi-simple and contains no involutions. Hence there is a minimal abelian connected normal subgroup $\Xi$ of $\Lambda$. From the Malcev-Iwasawa theorem we infer that $\Xi$ is either compact or homeomorphic (and then isomorphic) with some vector group $\mathbb{R}^{t}$. In the compact case, the identity component of $\Lambda$ acts trivially on $\Xi$, and $\operatorname{dim} \Lambda \leq 3$ by (7.4). If $\Xi \cong \mathbb{R}^{t}$, the same lemma yields that $t \leq 3$, and 
that the centralizer $C_{\Lambda}(P)$ of each one-parameter subgroup $P$ of $\Xi$ has dimension at most 3 . Now $\mathrm{C}_{\Lambda}(\mathrm{P})=\mathrm{C}_{\Lambda}(\rho)$ for each $\rho \in \mathrm{P} \backslash \mathbb{1}$, and $\operatorname{dim} \Lambda / \mathrm{C}_{\Lambda}(\rho) \leq t$, with equality holding only if $\Lambda$ acts transitively on $\Xi \backslash \mathbb{1}$. If $t=3$, then each group acting transitively on $\Xi \backslash \mathbb{1}$ contains a covering group of $\mathrm{SO}_{3} \mathrm{R}$ and thus an involution. This yields that $\operatorname{dim} \Lambda \leq \operatorname{dim} \mathrm{C}_{\Lambda}(\rho)+2 \leq 5$, and the theorem is proved.

Combining this with (6.8), we obtain:

(7.7) COROLLARY. Let $\Lambda$ be a semi-planar group of a 16-dimensional stable plane $M$. If a semi-simple subgroup $\Delta$ of $\Lambda$ leaves invariant some Baer subplane containing Fix $(\Lambda)$ (in particular, if $\Delta$ centralizes an involution $\alpha \in \Lambda$ ), then $\Delta$ is the product of at most two almost simple factors of dimension 3. If $\Delta$ is not almost simple, then at least one of the factors is isomorphic with $\mathrm{SU}_{2} \mathrm{C}$.

\section{SEMI-PLANAR GROUPS OF 16-DIMENSIONAL PLANES.}

In this section, let $\Lambda$ be a connected semi-planar group of a stable plane $\mathbf{M}=(M, \mathcal{M})$, where $\operatorname{dim} M=16$. Let $F=(F, \mathcal{F})$ with $F=\operatorname{Fix}(\Lambda)$, and let $(o, u, v)$ be a triangle in $F$.

(8.1) LEMMA. If there is a $\Lambda$-invariant Baer subplane $\mathbf{B}$ containing $\operatorname{Fix}(\Lambda)$, then $\operatorname{dim} \Lambda \leq 12$. If $\Lambda$ is a Lie group, this bound reduces to 8 .

Proof. The kernel $K$ of the restriction of $\Lambda$ to $B$ is compact by (6.1), and $\operatorname{dim} K \leq 7$. If $K$ is a Lie group, we have even $\operatorname{dim} K \leq 3$ by (6.8). According to (7.6), the group $\Lambda / K$ induced on $B$ is at most 5-dimensional.

(8.2) LEMMA. If there is no $\Lambda$-invariant Baer subplane containing Fix $(\Lambda)$, then for each $c \in M \backslash F$, the stabilizer $\Lambda_{c}$ is a Lie group.

Proof. According to (5.2), we have that $\left\langle F \cup c^{\Lambda}\right\rangle=\mathbf{M}$ for each point $c \in M \backslash F$. Let $\mathrm{Z}$ be the center of $\Lambda$. Since the stabilizer $Z_{c}$ acts trivially on $c^{\Lambda}$, we have that $Z_{c}=1$ and infer that $\Lambda_{c} \cong \Lambda_{c} / \mathrm{Z}_{c} \cong \Lambda_{c} \mathrm{Z} / \mathrm{Z}$ is a subgroup of the Lie group $\Lambda / \mathrm{Z}$.

(8.3) COROLLARY. If $\operatorname{dim} \Lambda>12$, then for each $c \in M \backslash F$ the stabilizer $\Lambda_{c}$ is a Lie group.

(8.4) LEMMA. Let $\Phi$ be a non-trivial, connected subgroup of $\Lambda$. Then $\operatorname{dim} C_{\Lambda}(\Phi) \leq 11$, or $\operatorname{dim} \Lambda=12$.

Proof. It suffices to consider the case where $\operatorname{dim} \Lambda>12$. Let $c$ be a point that is moved by $\Phi$. According to (8.3), the stabilizer $\Lambda_{c}$ is a Lie group. Since $\left(\mathrm{C}_{\Lambda}(\Phi)\right)_{c}$ acts trivially on $\left\langle F \cup c^{\Lambda}\right\rangle$, we conclude that $\operatorname{dim}\left(\mathrm{C}_{\Lambda}(\Phi)\right)_{c} \leq 3$ by $(6.8)$, and hence $\operatorname{dim} \mathrm{C}_{\Lambda}(\Phi) \leq 11$.

\section{Semi-simple groups.}

A locally compact connected group $\Delta$ is called semi-simple if it has no non-trivial closed connected abelian normal subgroup. In particular, the center of a semi-simple group has dimension 0 . If $\Delta$ is semi-simple and the factor group $\Delta / \mathrm{Z}(\Delta)$ is simple, then $\Delta$ is called almost simple. Each semi-simple group is a product of almost simple factors that centralize each other.

(8.5) LEMMA. The non-compact exceptional simple real Lie groups of type $\mathrm{G}_{2}(2)$ cannot act as semi-planar groups.

Proof. Such a group contains involutions whose centralizer is locally isomorphic with $\mathrm{SL}_{2} \mathrm{R} \times \mathrm{SL}_{2} \mathrm{R}$. This contradicts (7.7). 
(8.6) PROPOSITION. If $\Lambda$ is almost simple and $\operatorname{dim} \Lambda>10$, then $\Lambda$ is isomorphic with the compact exceptional simple Lie group of type $\mathrm{G}_{2}(-14)$.

Proof. Each simple group of type $A_{3}^{*}=D_{3}^{*}$ or $C_{3}^{*}$ contains a semi-simple compact lie group of rank 2, and the corresponding subgroups isomorphic with $\mathrm{SO}_{2} \mathrm{R} \times \mathrm{SO}_{2} \mathrm{R}$ have 3-dimensional centralizers. Each complex almost simple group of rank $\geq 2$ contains a subgroup isomorphic with $\mathbb{C}^{\times} \times \mathbb{C}^{\times}$, where $\mathbb{C}^{\times}$is the multiplicative group of the field of complex numbers. So (6.7) excludes the almost simple groups $\Lambda$ with $\operatorname{dim} \Lambda>10$, except the compact group of type $G_{2}(-14)$ and the non-compact groups of type $G_{2}(2)$. The latter cannot occur by $(8.5)$.

(8.7) LEMMA. If $\Lambda$ is semi-simple, but not almost simple, then there is a factor $\Psi$ of $\Lambda$ such that $\Psi / \mathrm{Z}(\Psi) \cong \mathrm{PSL}_{2} \mathrm{R}$, or $\Lambda$ is isomorphic with $\mathrm{SO}_{4} \mathrm{R}$.

Proof. Assume that there is no factor $\Psi$ with $\Psi / Z(\Psi) \cong \mathrm{PSL}_{2} \mathrm{R}$. Then each factor contains a subgroup that is locally isomorphic with $\mathrm{SU}_{2} \mathrm{C}$. Since these groups contain involutions, we obtain from (7.7) that each factor is locally isomorphic with $\mathrm{SU}_{2} \mathrm{C}$, and (6.13) yields the assertion.

(8.8) THEOREM. If $\Lambda$ is semi-simple, then $\Lambda \cong \mathrm{G}_{2}(-14)$, or $\operatorname{dim} \Lambda \leq 10$.

Proof. According to (7.7), (8.6) and (8.7), only the case remains where $\Lambda=\Sigma_{1} \Sigma_{2} \Sigma_{3} \Sigma_{4}$, where each factor $\Sigma_{i}$ is almost simple, and $\operatorname{dim} \Sigma_{i}=3$. According to (7.7), there is no $\Lambda$-invariant Baer subplane containing $F$, and $\Lambda_{c}$ is a Lie group for each $c \in M \backslash F$ by (8.2). Choose $c$ on a line of $F$ such that $c$ is moved by some one-parameter subgroup $\mathrm{P}$ in $\Sigma_{4}$, and put $\Psi=C_{\Lambda}(P)$. Then $\operatorname{dim} \Psi=10$, and $\operatorname{dim} \Psi_{c} \geq 2$. Since the identity component of $\Psi_{c}$ is a compact connected Lie group acting trivially on the Baer subplane generated by $F \cup c^{\mathrm{P}}$, we obtain that it is isomorphic with $\mathrm{SU}_{2} C$ by $(6.8)$. Hence at least one of the factors $\Sigma_{i}$ is compact and contains an involution $\alpha$. The centralizer of $\alpha$ in $\Lambda$ contains a semi-simple group with 3 almost simple factors in contradiction to (7.7).

\section{Groups that are not semi-simple.}

Assume now that $\Lambda$ is not semi-simple, and that $\operatorname{dim} \Lambda>12$. By definition of semisimplicity, there is a non-trivial connected abelian normal subgroup $\Xi$ of $\Lambda$. Choosing $\Xi$ minimal, we obtain from the Malcev-Iwasawa theorem that $\Xi$ is either compact or homeomorphic (and then isomorphic) with some vector group $\mathbb{R}^{t}$. If $\Xi$ is compact, the connected group $\Lambda$ acts trivially on $\Xi$, and $\operatorname{dim} \Lambda \leq 12$ by (8.4). Hence $\Xi \cong \mathbb{R}^{t}$, and by minimality $\Lambda$ acts irreducibly on $\Xi$ (via conjugation).

(8.9) LEMMA. Fix $(\Xi)=F$, and $\operatorname{dim} F \leq 4$.

Proof. If $\operatorname{dim} \operatorname{Fix}(\Xi)=8$, then $\Lambda$ leaves invariant a Baer subplane, and $\operatorname{dim} \Lambda \leq 12$ by (8.1), contrary to our hypotheses. If $\operatorname{dim}$ Fix $(\Xi) \leq 4$, then $\Lambda$ acts trivially on Fix $(\Xi)$ by (5.1), hence $\operatorname{Fix}(\Xi)=F$.

(8.10) LEMMA. The group $\Xi$ acts freely on $M \backslash F$, and $\operatorname{dim} \Xi \leq 7$.

Proof. For each $c \in M \backslash F$, the stabilizer $\Xi_{c}$ acts trivially on $\left\langle F \cup c^{\Xi}\right\rangle$. According to (5.2), this subplane is at least 8-dimensional, and $\Xi_{c}$ is compact by (6.1). But compact subgroups of $\Xi \cong \mathbb{R}^{t}$ are trivial, so $\Xi$ acts freely. The bound for the dimension of $\Xi$ follows from (3.10).

For the remainder of this section, choose $c \in o u \backslash F$, and let $\Gamma$ be the identity component of the stabilizer $\Lambda_{c}$. According to (8.3), $\Gamma$ is a Lie group. 
(8.11) LEMMA. If $\alpha$ and $\beta$ are two commuting involutions in $\Gamma$, then $\operatorname{dim} \mathrm{C}_{\Gamma}(\alpha) \leq 2$, or $\mathrm{C}_{\Gamma}(\alpha)$ contains a normal subgroup that is isomorphic with $\mathrm{SU}_{2} \mathrm{C}$, and $\operatorname{dim} \mathrm{C}_{\Gamma}(\alpha) \leq 4$.

Proof. According to (6.7), the involution $\beta$ induces a planar involution on $\mathrm{F}_{\alpha}$, and $\operatorname{Fix}(\alpha) \cap \operatorname{Fix}(\beta)$ carries a Baer subplane $D$ of $F_{\alpha}$. From [9: 1.34] (cf. (1.2a)) we obtain that $\operatorname{dim} F \geq 2$. Consequently, $D=\langle F \cup\{c\}\rangle$. Thus $\mathrm{C}_{\Gamma}(\alpha)$ acts trivially on $D$ and leaves invariant $\mathbb{F}_{\alpha}$. The kernel $\mathrm{K}$ of the restriction of $\mathrm{C}_{\Gamma}(\alpha)$ to $\mathrm{F}_{\alpha}$ is a compact Lie group, hence the identity component of $\mathrm{K}$ is isomorphic with $\mathrm{SO}_{2} \mathrm{R}$ or $\mathrm{SU}_{2} \mathrm{C}$ by (6.8). According to (6.3), a group of dimension $\leq 1$ is induced on $F_{\alpha}$.

(8.12) REMARK. Note that Lemma (8.11) does not hold in general: each stabilizer of a quadrangle in the Moufang plane over Cayley's octonions contains a subgroup isomorphic with $\mathrm{SO}_{4} \mathrm{R}$. The proof of the lemma shows, however, that this can only occur if the centralizer has a set of fixed points of dimension $\leq 2$.

Choose a minimal connected $\Gamma$-invariant subgroup $I I$ of $\Xi$ and a non-trivial element $\rho$ of $\Pi$. For the subgroup $P \cong \mathbb{R}$ generated by $\rho$, we have that $C_{\Gamma}(\rho)=C_{\Gamma}(P)$. This group acts trivially on $\left\langle F \cup c^{\mathrm{P}}\right\rangle$. Hence the identity component $\Psi$ of $\mathrm{C}_{\Gamma}(\mathrm{P})$ is a subgroup of $\mathrm{SU}_{2} C$ by (6.8), and $\operatorname{dim} \Gamma \leq \operatorname{dim} \mathrm{C}_{\Gamma}(\rho)+\operatorname{dim} \Pi \leq 3+t \leq 10$. On the other hand, we have that $\operatorname{dim} \Gamma=\operatorname{dim} \Lambda-\operatorname{dim} c^{\Lambda} \geq 13-8=5$. From these bounds we obtain that $\operatorname{dim} \Pi \geq \operatorname{dim} \Gamma-\operatorname{dim} \mathrm{C}_{\Gamma}(\rho) \geq 2$.

(8.13) LEMMA. $\operatorname{dim} \Pi \geq 4$.

Proof. Assume that $p=\operatorname{dim} \Pi \leq 3$. Then $\operatorname{dim} \mathrm{C}_{\Gamma}(\rho) \geq 5-3=2$. Thus the identity component $\Psi$ of $\mathrm{C}_{\Gamma}(\rho)$ is isomorphic with $\mathrm{SU}_{2} \mathrm{C}$. If the reductive group $\Psi$ acts nontrivially on $\Pi$, then $\Pi$ is the direct sum of $P$ and some $\Psi$-invariant subspace of dimension $\geq 3$. This contradicts the assumption that $p \leq 3$. Therefore $\Psi$ centralizes $\Pi$, and $\Gamma / \Psi$ acts almost effectively and irreducibly on $\Pi$.

If $p=3$ then $\Gamma / \Psi$ cannot be solvable. We obtain that $3=\operatorname{dim} \Gamma / \Psi=\operatorname{dim} \Gamma / \mathrm{C}_{\Gamma}(\rho)$. Therefore, $\Gamma$ acts transitively on $\Pi \backslash \mathbb{1}$. This yields that $\Gamma / \mathrm{C}_{\Gamma}(\Pi)$ contains a subgroup $\Sigma$ isomorphic with $\mathrm{SO}_{3} R$, which acts in the usual way on $\Pi \cong \mathbb{R}^{3}$. Now $\operatorname{dim} \Sigma / \mathrm{C}_{\Sigma}(\rho)=1$, in contradiction to the fact that $\operatorname{dim} \mathrm{C}_{\Gamma}(\rho)=3$.

There remains the case where $p=2$ and $\operatorname{dim} \Gamma=5$. Via the adjoint representation, the group $\Psi$ acts completely reducible on the Lie algebra $G$ of $\Gamma$. Let $C$ be a $\Psi$-invariant complement of the Lie subalgebra corresponding to $\Psi$. Then $\operatorname{dim} C \leq 2$, and $\Psi$ acts trivially on $C$. Consequently, the group $\Gamma$ is the product of $\Psi$ and the identity component $\Theta$ of $\mathrm{C}_{\Gamma}(\Psi)$. In particular, the central involution $\sigma$ of $\Psi$ lies in the center of $\Gamma$. Again, $\Theta$ acts transitively on $\Pi \backslash \mathbb{1}$. Since the centralizer $C_{\Gamma}(\rho)$ is compact, we obtain that $\Theta$ is isomorphic with the multiplicative group $\mathbb{C}^{\times}$of the field of complex numbers. Therefore, the 5-dimensional group $\Gamma$ contains commuting involutions and centralizes one of them. This contradicts (8.11).

(8.14) COROLLARY. The action of $\Gamma$ on $\mathrm{II}$ is effective.

Proof. We have that $\left\langle F \cup c^{\Pi}\right\rangle=\mathrm{M}$ by (7.4).

Any closed connected irreducible subgroup $\Delta$ of $\mathrm{GL}_{n} \mathbf{R}$ is the product of its semi-simple commutator group $\Delta^{\prime}$ and the identity component Z of its center (see [1: Chap. I, $\S 6$, no. 4, Proposition 5]). Applying Schur's Lemma, we obtain that $\mathrm{Z}$ is isomorphic with a subgroup of the multiplicative group $\mathbb{C}^{\times}$of the field of complex numbers. Therefore we have 
(8.15) COROLLARY. The commutator subgroup $\Gamma^{\prime}$ of $\Gamma$ is a semi-simple linear Lie group with $\operatorname{dim} \Gamma^{\prime} \geq 6$, and the identity component $\mathrm{Z}$ of the center of $\Gamma$ is isomorphic with a connected subgroup of $\mathbb{C}^{\times}$.

(8.16) LEMMA. The commutator group $\Gamma^{\prime}$ is almost simple.

Proof. Assume that $\Gamma^{\prime}=\Sigma_{1} \Sigma_{2}$ is not almost simple. Then each of the $\Sigma_{i}$ contains a subgroup isomorphic with $\mathrm{SO}_{2} \mathrm{R}$. Therefore there are commuting involutions, and $\Sigma_{i}$ is isomorphic with $\mathrm{SU}_{2} \mathrm{C}$ by (8.11). But now the central involution of $\Sigma_{1}$ lies in the center of $\Gamma^{\prime}$, in contradiction to (8.11).

(8.17) LEMMA. The commutator group $\Gamma^{\prime}$ is not isomorphic with $\mathrm{SO}_{3} \mathrm{C}$.

Proof. We assume that $\Gamma^{\prime}=\mathrm{SO}_{3} \mathrm{C}$, and consider the linear action of $\Gamma^{\prime}$ on $\Pi$ (via conjugation).

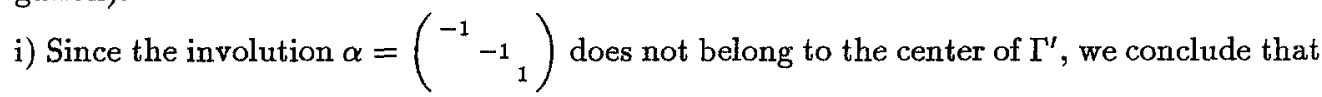
there is a non-trivial decomposition $\mathrm{II}=A \oplus A^{-}$, where $A=\mathrm{C}_{\Pi}(\alpha)$ and $\left.\alpha\right|_{A^{-}}=-1$. There are similar decompositions $\Pi=B \oplus B^{-}=C \oplus C^{-}$for $\beta=\left(\begin{array}{lll}{ }^{1} & & \\ & -1 & \\ & & -1\end{array}\right)$ and $\gamma=\alpha \beta$, since $\alpha, \beta, \gamma$ belong to the same conjugacy class. If $\mathrm{P}=A \cap B$ is not trivial, then the set $F \cup c^{\mathrm{P}}$ generates a Baer subplane (cf. (5.2)), and both $\alpha$ and $\beta$ act trivially on this subplane. According to (6.7), this is impossible, and we infer that $\mathrm{P}=\mathbb{1}$. Since $\alpha$ and $\beta$ commute, we conclude that we have the $\langle\alpha, \beta\rangle$-invariant direct decomposition $\Pi=A \oplus B \oplus C$, and $A, B, C$ are permuted transitively by certain elements of $\Gamma^{\prime}$ (recall that $\alpha, \beta, \gamma$ are conjugate). Now the inequality $4 \leq \operatorname{dim} \Pi=3 \cdot \operatorname{dim} A \leq 7$ yields that $\operatorname{dim} \Pi=6$.

ii) The centralizer $\mathrm{C}_{\Gamma}(\alpha)$ is the product of $\mathrm{Z}$ and the 2-dimensional group $\mathrm{C}_{\Gamma^{\prime}}(\alpha)$. Since the identity component of $\mathrm{C}_{\Gamma^{\prime}}(\alpha)$ is abelian, we conclude from (8.11) that $\operatorname{dim} \mathrm{C}_{\Gamma}(\alpha) \leq 2$. Therefore $Z=\mathbb{1}$.

iii) Let $\mathrm{N}$ be a compact normal subgroup of $\Lambda$ such that $\operatorname{dim} N=0$ and $\Lambda / \mathrm{N}$ is a Lie group. Via the adjoint representation, the reductive group $\Gamma / N$ acts completely reducibly on the Lie algebra $L$ of $\Lambda / \mathrm{N}$. This means that there is a (vector space-) decomposition $L=G \oplus P \oplus R$, where $G$ and $P$ are the Lie subalgebras corresponding to $\Gamma / \mathrm{N}$ and $\Pi / \mathrm{N}$, respectively. From $\operatorname{dim}(G \oplus P)=\operatorname{dim} \Gamma \Pi=12$ we infer that $R \neq 0$. Since $Z=\mathbb{1}$, the group $\Gamma / \mathrm{N}$ acts non-trivially on $R$, and $\operatorname{dim} R \geq 4$. Now $\operatorname{dim} \Lambda=\operatorname{dim} L \geq 16$, and $\operatorname{dim} c^{\Lambda}=\operatorname{dim} \Lambda-\operatorname{dim} \Gamma \geq 10$, a contradiction.

(8.18) LEMMA. The commutator group $\Gamma^{\prime}$ is isomorphic with $\mathrm{SU}_{3} \mathrm{C}$.

Proof. The group $\Gamma^{\prime}$ is a connected almost simple linear Lie group, and $6 \leq \operatorname{dim} \Gamma^{\prime} \leq 10$. According to (7.7), there is no central involution. The only groups with these properties are $\mathrm{SO}_{3} \mathrm{C} \cong \mathrm{PSL}_{2} \mathrm{C} \cong \Omega_{4}(1), \mathrm{SL}_{3} \mathrm{R}, \mathrm{SU}_{3} \mathrm{C}, \mathrm{PSU}_{3} \mathrm{C}, \mathrm{SU}_{3} \mathrm{C}(1), \mathrm{PSU}_{3} \mathrm{C}(1), \mathrm{SO}_{5} \mathrm{R}, \Omega_{5}(1)$ and $\Omega_{5}(2)$. The group $\mathrm{SO}_{3} \mathrm{C}$ has been excluded by (8.17). The group $\mathrm{SO}_{5} \mathbb{R}$ contains an involution which is centralized by $\mathrm{SO}_{4} \mathrm{R}$. Each of the non-compact groups in question contains a pair of commuting involutions such that one of them has a centralizer that does not fit the description in (8.11). Therefore all these groups are excluded, and only $\mathrm{SU}_{3} \mathrm{C}$ and $\mathrm{PSU}_{3} \mathrm{C}$ remain. Each linear representation of $\mathrm{PSU}_{3} \mathrm{C}$ has degree $\geq 8$. Thus, the assertion follows from (8.10).

(8.19) PROPOSITION. Let $\Lambda$ be a connected semi-planar group of a stable plane $M=$ $(M, \mathcal{M})$, where $\operatorname{dim} M=16$. If $\operatorname{dim} \Lambda>12$ then $\Lambda$ is semi-simple, or $\Lambda$ is isomorphic with 
$\mathrm{ASU}_{3} \mathrm{C}$ (i.e. the semi-direct product of $\mathrm{SU}_{3} \mathrm{C}$ with $\mathrm{C}^{3}$, where $\mathrm{SU}_{3} \mathrm{C}$ acts in the natural way).

Proof. Assume that $\Lambda$ is not semi-simple. There is a subgroup $\Upsilon \Pi$ of $\Lambda$ where $\Pi \cong R^{t}$, $4 \leq t \leq 7$, and $\Upsilon \cong \mathrm{SU}_{3} \mathrm{C}$. Each non-trivial linear representation of $\mathrm{SU}_{3} \mathrm{C}$ has degree $\geq 8$ or is equivalent to the natural representation on $C^{3}$. Therefore the action of $\Upsilon$ is equivalent to this representation. Let $\mathrm{Z}$ be the identity component of the centralizer $C_{\Lambda}(\Upsilon)$, and let $\Psi$ be a subgroup of $\Upsilon$ that is isomorphic with $T^{2}$. From (6.7) we infer that $Z=\mathbb{1}$. We consider the Lie group $\Lambda / N$ (for some compact normal subgroup $N$ such that $\operatorname{dim} N=0$ ). Via the adjoint representation, the semi-simple group $\Upsilon$ acts completely reducibly on the Lie algebra $L$ of $\Lambda / \mathrm{N}$ and leaves invariant the subalgebras $U$ and $P$ corresponding to $\Upsilon / \mathrm{N}$ and $\Pi / \mathrm{N}$, respectively. Now there is a $\Upsilon / \mathrm{N}$-invariant vector space $R$ such that $L$, considered as a vector space, is the direct $\operatorname{sum} U \oplus P \oplus R$. We infer that $\operatorname{dim} R \leq 2$. Consequently, $\Upsilon / \mathrm{N}$ acts trivially on $R$, and $R$ corresponds to $Z$. This yields that $R=0$ and $\Lambda=\Upsilon \Pi$.

(8.20) REMARK. Using a more elaborate compactness criterion (see [21]) H. Salzmann excluded the group $\mathrm{ASU}_{3} \mathrm{C}$ in the case of projective planes. Our attempts to generalize this criterion to the case of stable planes did not succeed, however.

We summarize our results.

(8.21) THEOREM. (Rigidity properties of 16-dimensional planes)

Let $\Lambda$ be a semi-planar group of a stable plane $\mathbf{M}=(M, \mathcal{M})$, where $\operatorname{dim} M=16$.

a) Either $\operatorname{dim} \Lambda \leq 12$, or the identity component of $\Lambda$ is isomorphic with the compact exceptional Lie group of type $\mathrm{G}_{2}(-14)$ or the group $\mathrm{ASU}_{3} \mathrm{C}$. These two groups are both 14-dimensional.

b) If $\operatorname{dim} \operatorname{Fix}(\Lambda)=8$, then $\Lambda$ is compact, and $\operatorname{dim} \Lambda \leq 7$.

c) If $\operatorname{dim} \operatorname{Fix}(\Lambda)=4$, then $\operatorname{dim} \Lambda \leq 11$.

Proof. i) Assertion b) follows from the compactness criterion (6.1) and (3.11).

ii) Assume the situation of $c$ ). If there is a $\Lambda$-invariant Baer subplane $B$, then $\Lambda$ induces a group of dimension $\leq 1$ on $\mathbf{B}$. The kernel of the restriction to $\mathbf{B}$ has dimension $\leq 7$ by b). Hence we may assume that there is no such subplane. Consequently, the stabilizer $\Lambda_{x}$ is a Lie group for each $x \in o u \backslash F i x(\Lambda)$. Now the set Fix $(\Lambda) \cup\{x\}$ generates a subplane $E$ of dimension 8 at least, and $\Lambda_{x}$ acts trivially on E. By (6.8), we conclude that $\operatorname{dim} \Lambda_{x} \leq 3$ and $\operatorname{dim} \Lambda \leq 11$.

\section{REFERENCES.}

[1] Bourbaki, N., Groupes et algèbres de Lie, Chapitre 1, Hermann, Paris, 1971.

[2] Bourbaki, N., Groupes et algèbres de Lie, Chapitre 9, Masson, Paris, 1982.

[3] Bredon, G.E., 'Transformation groups with orbits of uniform dimension', Michigan Math. J. 8 (1961) 139-147.

[4] Bredon, G.E., 'On homogeneous cohomology spheres', Ann. of Math. 73 (1961) 556565.

[5] Dold, A., Lectures on Algebraic Topology, Springer, Berlin etc., 1972.

[6] Dugundji, J., Topology, Allyn and Bacon, Boston etc., 1966.

[7] Grundhöfer, T., 'Automorphism groups of compact projective planes', Geom. Ded. 21 (1986) 291-298. 
[8] Halder, H.R., 'Die Dimension der Bahnen lokalkompakter Gruppen', Arch. der Math. 22 (1971) 302-303.

[9] Löwen, R., 'Vierdimensionale stabile Ebenen', Geom. Ded. 5 (1976) 239-294.

[10] Löwen, R., 'Halbeinfache Automorphismengruppen von vierdimensionalen stabilen Ebenen sind quasi-einfach', Math. Ann. 236 (1978) 15-28.

[11] Löwen, R., 'Topology and dimension of stable planes: On a conjecture by H. Freudenthal', J. Reine Angew. Math. 343 (1983) 108-122.

[12] Montgomery, D., Zippin, L., Topological transformation groups, Wiley, New York, 1955.

[13] Munkres, J.R., Topology: a first course, Prentice-Hall, Englewood Cliffs, New Jersey, 1975.

[14] Nagata, J., Modern Dimension Theory, Interscience, New York, 1965.

[15] Onishchik, A.L., Vinberg, E.B., Lie groups and algebraic groups, Springer, Berlin etc., 1990.

[16] Quinn, F., 'Resolutions of homology manifolds, and the topological characterization of manifolds', Invent. Math. 72 (1983) 267-284.

[17] Quinn, F., 'Corrigendum to Resolutions of homology manifolds, and the topological characterization of manifolds', Invent. Math. 85 (1986) 653.

[18] Quinn, F., 'An obstruction to the resolution of homology manifolds', Michigan Math. J. 34 (1987) 285-291.

[19] Richardson, R.W., 'Groups acting on the 4-sphere', Illinois J. Math. 5 (1961) 474-485.

[20] Salzmann, H., 'Compact 8-dimensional projective planes with large collineation groups', Geom. Ded. 8 (1979) 139-161.

[21] Salzmann, H., 'Automorphismengruppen 8-dimensionaler Ternärkörper', Math. Z. 166 (1979) 265-275.

[22] Smith, P.A., 'New results and old problems in finite transformation groups', Bull. Am. Math. Soc. 66 (1960) 401-415.

[23] Stroppel, M., Achtdimensionale stabile Ebenen mit quasieinfacher Automorphismengruppe, Dissertation, Tübingen, 1991.

[24] Szenthe, J., 'On the topological characterization of transitive Lie group actions', Acta Scient. Math. Szeged 36 (1974) 323-344.

[25] Tits, J., Tabellen zu den einfachen Lie Gruppen und ihren Darstellungen, Lect. Notes in Math. 40, Springer, Berlin etc., 1967.

[26] Varadarajan, V.S., Lie groups, Lie algebras, and their representations, Springer, Berlin etc., 1984.

Markus Stroppel

Fachbereich Mathematik

Technische Hochschule Darmstadt

Schloßgartenstr. 7

D-6100 Darmstadt 